\title{
Dystrophin Is Required for Appropriate Retrograde Control of Neurotransmitter Release at the Drosophila Neuromuscular Junction
}

\author{
Mariska C. van der Plas, ${ }^{1 \star}$ Gonneke S. K. Pilgram, ${ }^{1 \star}$ Jaap J. Plomp, ${ }^{2,3}$ Anja de Jong, ${ }^{1}$ Lee G. Fradkin, ${ }^{1}$ and \\ Jasprina N. Noordermeer ${ }^{1}$ \\ ${ }^{1}$ Laboratory of Developmental Neurobiology, ${ }^{2}$ Section of Neurophysiology, Department of Molecular and Cell Biology, and ${ }^{3}$ Department of Neurology, \\ Leiden University Medical Center, 2300 RC Leiden, The Netherlands
}

\begin{abstract}
Mutations in the human dystrophin gene cause the Duchenne and Becker muscular dystrophies. The Dystrophin protein provides a structural link between the muscle cytoskeleton and extracellular matrix to maintain muscle integrity. Recently, Dystrophin has also been found to act as a scaffold for several signaling molecules, but the roles of dystrophin-mediated signaling pathways remain unknown. To further our understanding of this aspect of the function of dystrophin, we have generated Drosophila mutants that lack the large dystrophin isoforms and analyzed their role in synapse function at the neuromuscular junction. In expression and rescue studies, we show that lack of the large dystrophin isoforms in the postsynaptic muscle cell leads to elevated evoked neurotransmitter release from the presynaptic apparatus. Overall synapse size, the size of the readily releasable vesicle pool as assessed with hypertonic shock, and the number of presynaptic neurotransmitter release sites (active zones) are not changed in the mutants. Short-term synaptic facilitation of evoked transmitter release is decreased in the mutants, suggesting that the absence of $d y s t r o p h i n$ results in increased probability of release. Absence of the large dystrophin isoforms does not lead to changes in muscle cell morphology or alterations in the postsynaptic electrical response to spontaneously released neurotransmitter. Therefore, postsynaptic glutamate receptor function does not appear to be affected. Our results indicate that the postsynaptically localized scaffolding protein Dystrophin is required for appropriate control of neuromuscular synaptic homeostasis.
\end{abstract}

Key words: Dystrophin; neuromuscular junction; neurotransmitter release; Drosophila; DGC; synaptic homeostasis

\section{Introduction}

Duchenne (DMD) and Becker muscular dystrophy are caused by mutations in the dystrophin (dys) gene (Hoffman et al., 1987). Dystrophin and its partially redundant homolog Utrophin are associated with a number of other proteins, members of the Dystrophin-glycoprotein complex (DGC) (Ervasti and Campbell, 1991). The DGC links the actin cytoskeleton to the extracellular basal lamina, providing tensile strength to muscle fibers. In addition to its structural role, the DGC acts to scaffold signaling molecules (for review, see Rando, 2001). How the DGC and associ-

\footnotetext{
Received Sept. 25, 2005; revised Nov. 8, 2005; accepted Nov. 9, 2005.

This work was supported by Pionier and Aspasia grants from the "Nederlandse Organisatie voor Wetenschappelijk Onderzoek." We thank Aaron DiAntonio, Peter ten Dijke, Corey Goodman, Leslie Griffith, David Gubb, Mark Kelley, Brian McCabe, Mike O'Connor, Kai Zinn, and the Bloomington and Kyoto stock centers for fly stocks and antibodies; Mieke Mommaas, Rick Fetter, David Featherstone, Gert-Jan van Ommen, Johan den Dunnen, and Irena Miguel-Aliaga for advice; and Monique Radjkoemar-Bansraj, Rene Wouda, Linda Dekkers, and Simon Kaja for their help with the experiments. We also acknowledge Grae Davis, Gert-Jan van Ommen, Pascale Dijkers, Aaron DiAntonio, Richard Baines, and two anonymous reviewers for providing insightful suggestions on this manuscript.

${ }^{*}$ M.C.v.d.P. and G.S.K.P. contributed equally to this work.

Correspondence should be addressed to either Lee G. Fradkin or Jasprina N. Noordermeer, Laboratory of Developmental Neurobiology, Leiden University Medical Center, Wassenaarseweg 72, 2300 RC Leiden, The Netherlands. E-mail: l.g.fradkin@lumc.nl or j.n.noordermeer@lumc.nl.

DOI:10.1523/JNEUROSCI.4069-05.2006

Copyright $\odot 2006$ Society for Neuroscience $\quad$ 0270-6474/06/260333-12\$15.00/0
}

ated signaling partners interact with other cellular pathways remains unclear.

Members of the mammalian DGC complex, including Dystrophin and Utrophin, are found in the CNS and the extrasynaptic and postsynaptic regions of muscle cells (for review, see Blake et al., 2002). Reduction of postsynaptic junctional folds and acetylcholine receptor (AChR) clustering are observed at the neuromuscular junction (NMJ) in the dystrophin/utrophin double knock-out mouse, but electrophysiological analyses revealed a normal postsynaptic response to spontaneous neurotransmitter release (Deconinck et al., 1997a; Grady et al., 1997). These and other data suggest that dystrophin and utrophin likely play partially redundant and subtle roles at the mammalian NMJ (Lyons and Slater, 1991; Deconinck et al., 1997b). A single dystrophin ortholog exists in Drosophila (Greener and Roberts, 2000); its isoforms are predominantly expressed in the muscle and nervous system (Neuman et al., 2001; Dekkers et al., 2004). In this study, we investigate the role of the postsynaptically localized dystrophinlike protein 2 (DLP2) Dystrophin isoform at the Drosophila NMJ.

During maturation and modification of synaptic contacts, homeostatic mechanisms match neurotransmitter release levels to changing postsynaptic requirements, keeping depolarization levels within a narrow range (for review, see Turrigiano, 1999; Davis and Bezprozvanny, 2001). Studies of myasthenia gravis patient 
NMJs (in which autoantibodies reduce AChR number) revealed that, in addition to anterograde signaling, the homeostatic machinery encompasses retrograde signals required for the appropriate regulation of presynaptic neurotransmitter release (CullCandy et al., 1980; Plomp et al., 1995). Similar compensatory neurotransmitter release upregulation was shown in rodents with decreased postsynaptic AChR levels (Plomp et al., 1992; Sandrock et al., 1997) and in Drosophila with reduced DGluRIIA glutamate receptor function (Petersen et al., 1997; Davis et al., 1998; DiAntonio et al., 1999).

In Drosophila, several proteins have been implicated in NMJ retrograde signaling (Keshishian and Kim, 2004), including members of the bone morphogenetic protein (BMP) pathway, the presynaptic type II receptor wit (for wishful thinking), and its muscle-derived ligand gbb (for glass bottom boat) (Aberle et al., 2002; Marques et al., 2002; McCabe et al., 2003). Postsynaptic calcium/calmodulin-dependent kinase (CaMKII) activity has also been reported to modulate retrograde signaling via wit (Haghighi et al., 2003).

Here, we take advantage of Drosophila genetics to study the role of $d y$ strophin at the NMJ. We show that $d y$ strophin is required for appropriate homeostatic control of neurotransmitter release. Absence of postsynaptically localized Dystrophin results in a wit-dependent increase in neurotransmitter release, leading to enhanced muscle depolarization. Furthermore, short-term synaptic facilitation is impaired in the dystrophin mutant, indicating an increase in the probability of release. These results reveal a novel role for $d y$ strophin in the dynamics of neurotransmitter release.

\section{Materials and Methods}

Fly stocks. $w^{1118}$, the genetic background in which the dystrophin mutations were generated, and $d y s^{E 31}$, a precise excision of the P-element used for the mutagenesis, served as the wild-type controls for stainings, electrophysiology, and EM analyses. The following Gal4 driver lines were used: 24B-Gal4 (Brand and Perrimon, 1993), G14-Gal4 (Aberle et al., 2002), Elav-Gal4 (Luo et al., 1994), and OK6-Gal4 (Aberle et al., 2002). The GS12472 insertion (Toba et al., 1999), $1.9 \mathrm{~kb}$ upstream of the DLP2 ATG initiator, was used to overexpress DLP2. Upstream activating sequence lines (UAS)-Ala and UAS-CaMKII ${ }^{\text {T287D }}$ (Griffith et al., 1993; Jin et al., 1998) were used to inhibit CaMKII or to overexpress the constitutively active form of CaMKII, respectively. The wit ${ }^{A 12}$ and wit ${ }^{B 11}$ alleles were used in combination to generate wit hypomorphic larvae or embryos (Marques et al., 2002). Green fluorescent protein or Tubby balancers were used to identify homozygous individuals.

Generation of dystrophin mutants and transgenic constructs and quantitative PCR analyses. The EP3397 insert (Rorth, 1996), 750 bp upstream of the DLP2 initiator ATG, was used in a P-element mobilization screen to create deletions in the dystrophin gene (Tower et al., 1993). Mutants that lack specific dystrophin isoforms were identified. $d y s^{E 6}$, that has a deletion of $2.7 \mathrm{~kb}$ and a precise excision, $d y s^{E 31}$, are described in this paper. Sequence analysis indicates that the deletion in $d y s^{E 6}$ removes genomic sequences encoding all of the DLP2 $5^{\prime}$ untranslated region and extends $\sim 200$ bp downstream of the DLP2 initiator ATG but does not delete the unique DLP1 first exon. The CG6255 gene, which encodes a putative testis-specific succinate-coenzyme A ( CoA) ligase activity and is present in the intron between the DLP1 and DLP2 first exons, is deleted in $d y s^{E 6}$. An independently derived mutation, the GE20705 P-element insertion (obtained from GenExel, Daejeon, South Korea) is present 250 bp upstream of the Dp186 initiator ATG and is referred to as $d y s^{G E 20705}$. A transgenic RNA interference (RNAi) construct was made to reduce the expression of the large dystrophin isoform DLP2 in a tissue-specific manner using the UAS-Gal4 expression system (Brand and Perrimon, 1993). The RNAi-dys $\mathrm{NH}_{2}$ transgene contains sequences specific to the $d y$ strophin large isoform mRNAs (base pairs 610-1532 of GenBank accession number AF297644) in a pUAST (Brand and Perrimon, 1993) derivative bearing the $m u b$ intron (Reichhart et al., 2002) between the gene regionspecific repeats to increase the efficiency of RNA interference. Multiple independent transgenic lines of this RNA-interference construct were derived; two were shown to yield identical results (data not shown).

To determine the effects of the $d y s^{E 6}$ and $d y s^{G E 20705}$ mutations and expression of the RNAi-dys $\mathrm{NH}_{2}$ transgene on the expression levels of the dystrophin isoforms and CG6255, we performed semiquantitative reverse transcription (RT)-PCR on total RNA derived from larval body walls (DLP2), total larva (DLP1 and CG6255) or larval brain-imaginal disc complexes (Dp186) using primer pairs specific for DLP1, DLP2, Dp186, and CG6255. A primer set specific for the ribosomal protein RP49 was used to evaluate total mRNA levels in the samples. These primers were chosen to generate intron-spanning products to allow discrimination of cDNA-derived versus genomic DNA-derived PCR products. Primers used were as follows: DLP1 forward, ATGCACGATCCCAGTGACTACG; DLP1 reverse, TTGGCATTGCCACCCACT; DLP2 forward, CGTAAAGACTTGAAACGCGTCG; DLP2 reverse, TGGATTCCATGGCGTGGT; Dp186 forward, GCTGCTGGTGGTGGTGGTGGAA; DP186 reverse, ATTGGCCTCGCCCACGGATG; CG6255 forward, AATCGAGGAAGCAAGATGCTCC; CG6255 reverse, GCCTCGCAGCCAGTGTTCTTCT; RP49 forward, ATGACCATCCGCCCAGCA; and RP49 reverse, TTGGGGTTGGTGAGGCGGAC. Total mRNA was prepared from tissues using the RNeasy kit (Qiagen, Hilden, Germany), including a DNAase treatment to reduce potential genomic DNA contamination. Reverse transcriptions were performed with the Superscript First-Strand Synthesis System kit (Invitrogen, Breda, The Netherlands) according to the instructions supplied. cDNA samples were adjusted to equivalent A260 values, and either twofold or threefold dilutions (as indicated in the legend of supplemental Fig. 2, available at www. jneurosci.org as supplemental material) were performed to ensure that the assay was in a range in which the product band intensity corresponded to input total cDNA. All samples within a set were treated identically; those used for RP49 RT-PCR were first diluted 80 -fold because of the relatively higher abundance of RP49 mRNA. Standard 30 cycle PCR was performed using a Taq derivative thermostable polymerase, and equivalent volumes of reaction products were electrophoresed and ethidium bromide stained. Equivalently intense bands in the linear range of the assay were identified in the mutant and transgenic RNAiexpressing titrations and compared with the wild-type titration to estimate fold reductions in expression.

Generation of polyclonal antibodies, immunohistochemistry, RNA in situ hybridization, and Western blotting. Three rabbit antisera were raised against three His-tagged Dystrophin antigens: (1) anti-Dyslarge, which is large-isoform specific (amino acids 406-733 of the DLP2 protein, GenBank accession number AAK15256), (2) anti-Dp186, which is Dp186 specific (amino acids 15-170 of GenBank accession number AAK15257), and (3) the pan-Dystrophin isoform-specific anti-dys $\mathrm{CO}_{2} \mathrm{H}$ (amino acids 3037-3223 of the DLP2 protein, GenBank accession number AAK15256). Immunohistochemistry and RNA in situ hybridizations using an antisense probe containing exon 4 sequences common to all large dystrophin isoforms were done as described previously (Dekkers et al., 2004). The 368 bp DLP1-specific and 421 bp DLP2-specific probes were derived by RT-PCR of first-strand embryonic cDNA and correspond to base pairs 55321-55688 and 58724-59059 of a chromosome 3R region $92 \mathrm{~A}$ bacterial artificial chromosome clone (GenBank accession number AC008192). Anti-dysCO ${ }_{2} \mathrm{H}$ (1:3000), anti-Dyslarge (1:3000), anti-HRP (1:500) (Promega, Madison, WI), anti-DGluRIIA (1:500) (Developmental Studies Hybridoma Bank, University of Iowa, Iowa City, IA) (Schuster et al., 1991), anti-DGluRIIB (1:2500) (Marrus et al., 2004), anti-Discslarge (Developmental Studies Hybridoma Bank) (Parnas et al., 2001) (1:500), anti-actin (1:5000) (MP Biomedicals, Aurora, OH), antiPhospho-Mad (Tanimoto et al., 2000) (1:1000), Alexa Fluor-conjugated secondary antibodies (Invitrogen, Breda, The Netherlands), and an antiribosomal subunit PS3 antibody were used as described. Standard epifluorescence and confocal microscopy were used to visualize the samples.

Electrophysiology and statistical analyses. Intracellular microelectrode electrophysiological recordings were performed at muscle 6 in segments A3-A5 using dissected third-instar female larvae bathed in HL3 containing the indicated $\mathrm{Ca}^{2+}$ concentrations. Initial characterization of the 
A)

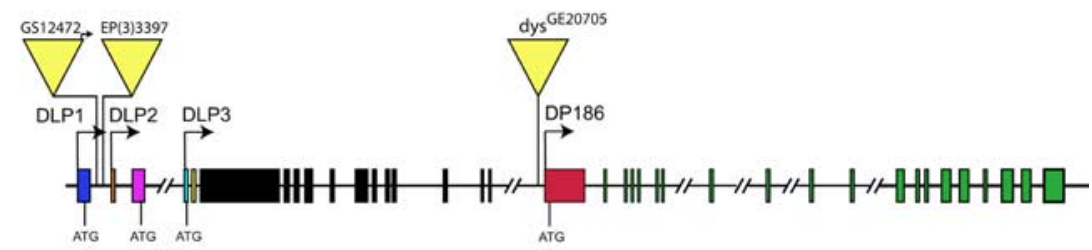

$2.7 \mathrm{~kb} \square$ deletion in dys ${ }^{\mathrm{E}} \mathrm{C}$

B)

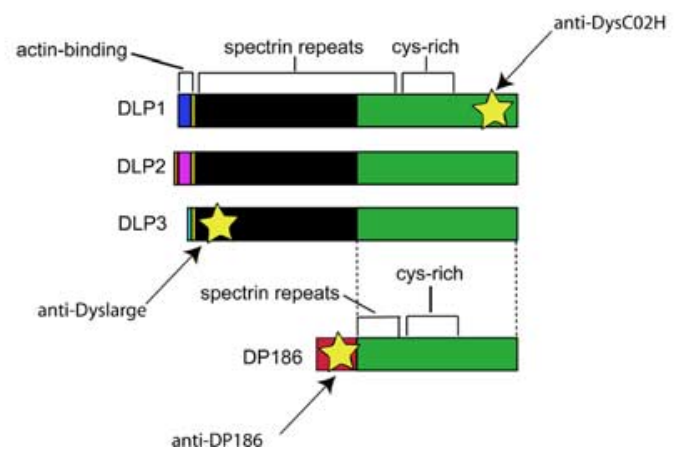

Figure 1. dystrophin gene structure, the position of the P-elements, and deletion in the dys ${ }^{E 6}$ mutant and the protein regions used for antibody production. $\boldsymbol{A}$, There are four known dystrophin isoforms, the large isoforms DLP1, DLP2, and DLP3 and the short Dp186 isoform. The EP3397 P-element insertion was used to generate the large-isoform mutants, and the GS12472 P-element insertion was used in combination with Gal4 drivers to express DLP2. The position of the dys ${ }^{E 6} 2.7 \mathrm{~kb}$ deletion is indicated. The GE20705 P-element insertion near the Dp186 initiator ATG reduces expression of both Dp186 and the large isoforms. Exons are indicated as bars and introns as horizontal lines. $\boldsymbol{B}$, The conserved Dystrophin protein domains, an actin-binding domain, spectrin repeats, and a cysteine (cys)-rich (-terminal domain are indicated for DLP1, DLP2, DLP3, and Dp186. Dp186 has a unique $\mathrm{N}$-terminal domain. Regions used to generate the anti-Dyslarge, anti-Dys $\mathrm{CO}_{2} \mathrm{H}$, and anti-Dp186 antisera are indicated.

described previously (Suzuki et al., 2002), and the increased $\mathrm{mEJP}$ frequency was measured for $1 \mathrm{~min}$ after the start of the incubation. In some control experiments, we monitored the sucrose response for 5-20 $\mathrm{min}$ and observed that the elevated $\mathrm{mEJP}$ frequency declines only very slowly during the first 5-20 min after the start of the incubation ( $\sim 50 \%$ per $10 \mathrm{~min})$. This contrasts with the burst-like, only-seconds-lasting, response observed in Drosophila embryonic NMJs (Suzuki et al., 2002; Kidokoro et al., 2004). Third-instar larval Drosophila NMJs in this respect resemble frog and mouse NMJs, in which the sucrose response also remains active for many minutes (Grinnell et al., 2003) (J. J. Plomp, unpublished observations).

NMJs at muscles 6 of three separate segments were measured for each larva, and, for each parameter, the average larval value was calculated and taken into the calculation of the group mean values; the number of larvae ( $n$ value) per genotype is indicated in the figure legends. ANOVA statistics were performed (SPSS version 11; SPSS, Chicago, IL), and differences were considered significant when $p<0.05$.

Transmission electron microscopy and morphometric analysis. Previously described techniques for dissection, fixation, embedding, and sectioning were followed (Lin and Goodman, 1994). Complete semi-serial sections of muscles 6 and 7, segment A3-A5, were cut, and electron micrographs were made of type Ib boutons using a CM10 electron microscope (Philips, Amsterdam, The Netherlands). Within a bouton, the micrograph with the largest diameter of the

mutants was performed at $1.5 \mathrm{~mm} \mathrm{Ca}^{2+}$ to approximate physiological conditions. However, because the extent of increased transmitter release at $d y$ strophin mutant NMJs did not show $\mathrm{Ca}^{2+}$ dependence (see Fig. 5A), we performed the remainder of the measurements at $0.6 \mathrm{mM} \mathrm{Ca}^{2+}$ to prevent occasional muscle contractions. Electrical input resistance of muscle fibers was similar for all genotypes $(\sim 4.5 \mathrm{M} \Omega$; data not shown). Synaptic signals were only measured when the resting membrane potential was below $-50 \mathrm{mV}$ (on average, the membrane potential was -60 $\mathrm{mV}$ in all samples, which is in accordance with the use of HL3 buffer) and recorded using a Geneclamp 500B amplifier (Axon Instruments, Union City, CA), low-pass filtered at $10 \mathrm{kHz}$, high-pass filtered at $0.5 \mathrm{~Hz}$, and digitized using a Digidata 1322A and pClamp9 software (Axon Instruments). The miniature excitatory junction potentials (mEJPs) were recorded continuously for $1 \mathrm{~min}$. During supramaximal stimulation of the ventral nerve cord via a suction electrode connected to a pulse generator, 30 EJPs were recorded at $0.3 \mathrm{~Hz}$ stimulation. Raw mEJP data were first filtered off-line in Clampfit 9.0 (Axon Instruments) using a boxcar filter with 19 smoothing points. Subsequently, the mean mEJP amplitude and frequency were determined using the peak detection feature of MiniAnalysis 6.0 (Synaptosoft, Decatur, GA); all events were verified by eye. EJP amplitudes were analyzed using Clampfit 9.0. All amplitudes were normalized to a membrane potential of $-60 \mathrm{mV}$, because all of the measurements were performed in the linear range of the EJP amplitude $-V_{\mathrm{m}}$ curve (Jan and Jan, 1976). Quantal content (QC) was calculated per NMJ by dividing the mean EJP amplitude (calculated from 30 events) by the mean mEJP amplitude (calculated from $\sim 100$ events). The EJP amplitude was first corrected for nonlinear summation (Martin, 1955) using an $f$ factor of 0.4 (B. A. Stewart, personal communication) and a reversal potential of $-10 \mathrm{mV}$. Failure analysis was performed in $0.25 \mathrm{~mm}$ $\mathrm{Ca}^{2+}$, and QC was calculated using the formula $m=\ln \left(N / N_{0}\right)$, where $m$ is the QC, $N$ is the number of stimuli, and $N_{0}$ is the number of failures. QC estimated using the variance method was calculated using the formula $m=(\text { mean EJP/SD })^{2}$. Paired-pulse facilitation of EJP amplitude was assessed using $50 \mathrm{~ms}$ interstimulus interval in $0.6 \mathrm{mM} \mathrm{Ca}^{2+}$. Hypertonic shock was induced by adding $50 \mathrm{~mm}$ sucrose to the HL3 medium as bouton, corresponding to the bouton midline, was selected for analysis. Fifteen electron micrographs from three larvae of each genotype were scanned, and the morphometric and statistical analyses were performed using Scion (Frederick, MD) software and SPSS version 11, respectively.

Muscle area, terminal length, and bouton number were determined at muscles 6 and 7 of segment A3 from digital images captured by light and fluorescence microscopy and analyzed using Scion software. Anti-HRP (Jan and Jan, 1982) was used to visualize the boutons. Thirty segments from 15 individual larvae from each genotype were measured.

\section{Results}

The large Dystrophin isoform DLP2 is expressed at the NMJ postsynaptic region

The Drosophila dystrophin gene encodes at least four protein isoforms bearing a number of highly conserved domains (Fig. 1) (Greener and Roberts, 2000; Neuman et al., 2001). The three large isoforms DLP1, DLP2, and DLP3 have an N-terminal actinbinding domain, spectrin repeats, and a C-terminal cysteine-rich domain, which in mammals has been shown to interact with other DGC proteins. A shorter isoform, Dp186, has a unique $\mathrm{N}$-terminal domain appended to the pan-Dystrophin C-terminal domain (Fig. 1).

DLP1, DLP2, and Dp186 are expressed during all stages of development, whereas DLP3 is only expressed in the adult fly (Neuman et al., 2001; Dekkers et al., 2004). During embryogenesis, the DLP1 mRNA is present in the visceral mesoderm, whereas DLP2 is expressed in the visceral mesoderm, in muscle attachment sites, throughout muscle fibers, in the mesectodermal cells at the midline, and the gut (supplemental Fig. 1, available at www.jneurosci.org as supplemental material) (Dekkers et al., 2004). Dp186 is highly expressed in the embryonic CNS but absent from the musculature. In larvae, the large dystrophin iso- 
form DLP2 mRNA, but not DLP1, is found in all muscle fibers (supplemental Fig. 1, available at www.jneurosci.org as supplemental material). The large isoforms are not detectably expressed in the larval brain or neuropil (supplemental Fig. 1, available at www.jneurosci.org as supplemental material) (Fig. $2 H$ ). In contrast, Dp186 mRNA is found most predominantly throughout the larval neuropil and brain and in the eyeantennal discs (supplemental Fig. 1, available at www.jneurosci.org as supplemental material).

We have generated three regionspecific antibodies that recognize (1) the known large Dystrophin isoforms DLP1, DLP2, and DLP3 (anti-Dyslarge), (2) the CNS-specific isoform (anti-Dp186), and (3) the C-terminal region common to all Dystrophin isoforms (anti-DysCO ${ }_{2} \mathrm{H}$ ) (Fig. 1B). Western analyses of embryonic extracts using these antibodies show that the anti-Dyslarge antibodies recognizes a protein of the expected size for the large isoforms $(\sim 400 \mathrm{kDa})$, whose level is strongly reduced in the dystrophin mutant $d y s^{G E 20705}$ and increased when the large isoform DLP2 is overexpressed. The anti-Dp186 antibody recognizes a protein species of the appropriate size $(\sim 190 \mathrm{kDa})$, and the anti-DysCO ${ }_{2} \mathrm{H}$ antibody predominantly recognizes the large isoforms, but also Dp186, when overexpressed (Fig. 2I).

The region-specific antibodies were used to determine the protein expression domains of the Dystrophin isoforms. The anti-DysCO $\mathrm{C}_{2} \mathrm{H}$ (Fig. 2A) and antiDyslarge (Fig. $2 D$ ) antibodies label synaptic and extrasynaptic sites of expression in third-instar larval body walls. To determine whether the Dystrophin protein is present at the presynaptic or the postsynaptic side of the NMJ, larval body walls were colabeled for the presynaptically localized HRP protein (Jan and Jan, 1982) and the Dystrophin protein. No overlap in staining was apparent (Fig. $2 F$ ), indicating that the Dystrophin protein is postsynaptically localized at the larval NMJ. This result is consistent with our finding that the large dystrophin mRNAs are absent from the neuropil in which the motoneuron cell bodies are located (Fig. $2 H$ ). The extrasynaptic Dystrophin protein colocalizes with actin at the I-band of sarcomeres (Fig. 2G). Synaptic and extrasynaptic Dystrophin expression levels are severely reduced in the dystrophin mutant $d y s^{G E 20705}$, indicating the specificity of the antibody (Fig. $2 B, I)$. When the DLP2 large Dystrophin isoform is overexpressed in muscle using the Gal4-UAS yeast transcriptional activation system (Brand and Perrimon, 1993) (Materials and Methods), Dystrophin protein accumulates highly at the postsynaptic regions of the $\mathrm{NMJ}$ (Fig. 2C,E). These results indicate that the large Dystrophin isoform DLP2 is localized to both the postsynaptic region of the NMJ and extrasynaptic regions, in which it colocalizes with actin.

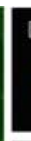
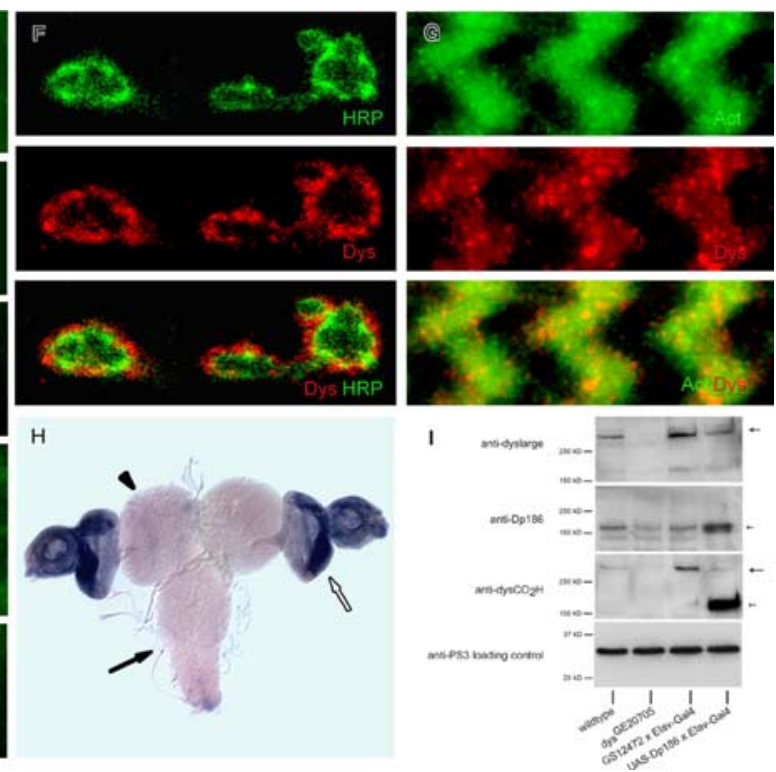

Figure 2. The large Dystrophin isoforms are localized postsynaptically. Third-instar larval body walls were stained with antiDys $\mathrm{C}_{2} \mathrm{H}$, which recognizes all Dystrophin isoforms $(\boldsymbol{A}-\boldsymbol{C})$, anti-Dyslarge, which recognizes only the large isoforms $(\boldsymbol{D}, \boldsymbol{E})$, double expressed at the wild-type third-instar larval NMJ at synaptic and extrasynaptic sites. B, Dystrophin protein is severely reduced in dys ${ }^{G E 20705}$ mutant. C, The DLP2 isoform protein accumulates highly at the NMJ after overexpression in the muscle (G14Dys Dyslarge. $\boldsymbol{E}$, Overexpressed DLP2 (G14-Gal4/+; GS12472/+) can also be visualized using anti-Dyslarge antisera. $\boldsymbol{F}$, Double

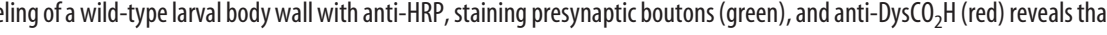

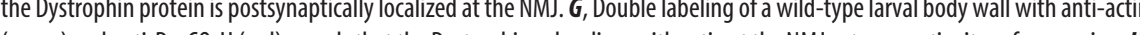
green) and anti-Dys $\mathrm{CO}_{2} \mathrm{H}$ (red) reveals that the Dystrophin colocalizes with actin at the NMJ extrasynaptic sites of expression. $\boldsymbol{H}$

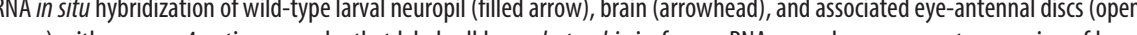
dystrophin isoform mRNAs in the neuropil or brain. I, Western blot analysis of embryo extracts prepared from wild-type, dys $^{\text {GE20705 }}$, Elav-Gal4/GS12472 (overproducing DLP2), and Elav-Gal4/UAS-Dp186 (overproducing Dp186) using the indicated antibodies. The large Dystrophin isoforms (anti-Dyslarge panel) are absent from the mutant dys ${ }^{G E 20705}$ and overexpressed in the

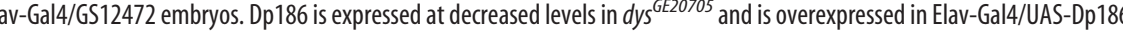
and Dp186 in the overproducing embryos. The arrows indicate the large Dystrophin isoforms, and the short arrows indicate the Dp186 isoform. All lanes were loaded equally, as confirmed by the anti-ribosomal subunit PS3 antibody (anti-PS3 panel), except the last lane of the Dp186 blot, in which 5\% of the protein was loaded to permit comparison of the extracts in a single exposure.

\section{Lack of postsynaptic Dystrophin results in an increase in neurotransmitter release}

To investigate the roles of dystrophin during Drosophila development, we have generated stocks that lack, or have severely reduced, expression of the large Dystrophin isoforms by P-element excision mutagenesis. The mutant $d y s^{E 6}$ has a deletion of $2.7 \mathrm{~kb}$ (Fig. $1 A) ; d y s^{E 31}$ is a precise excision control of the EP3397 P-element. We also used the independently derived P-element line $d y s^{G E 20705}$ mentioned above, which is inserted 250 bp upstream of the Dp186 initiator codon. To determine whether, and to what extent, the mRNA levels of the specific Dystrophin isoforms in these mutants are affected, we performed semiquantitative RT-PCR on total RNA derived from mutant larval tissues using primers specific for each of the isoforms (see Material and Methods) (supplemental Fig. 2, available at www.jneurosci.org as supplemental material). Dystrophin isoform proteins were proteolytically degraded during the preparation of larval extracts, thus precluding analysis of their expression levels by Western blotting. RT-PCR analyses revealed that $d y s^{E 6}$ expresses DLP1 mRNA at wild-type levels but lacks DLP2 mRNA, whereas $d y s^{G E 20705}$ shows an approximate threefold reduction in DLP1 and an approximate fourfold reduction in DLP2 mRNA levels. 
Dp186 mRNA levels are wild type in $d y s^{E 6}$ but are significantly reduced $\left(\sim 27\right.$-fold) in $d y s^{G E 20705}$. The precise excision control $d y s^{E 31}$ shows wild-type expression levels of all isoforms. The lack of detectable large-isoform expression in the $d y s^{G E 20705}$ mutant larval musculature was confirmed by staining mutant body walls with the anti-Dys $\mathrm{CO}_{2} \mathrm{H}$ antibody (Fig. $2 \mathrm{~B}$ ) and by Western blot analysis of embryonic extracts using the three region-specific antibodies (Fig. 2I). The CG6255 gene, which encodes a putative testis-specific succinate-CoA ligase and is located in the intron between the DLP1 and DLP2 first exons, is deleted in $d y s^{E 6}$ but not in $d y s^{G E 20705}$. RT-PCR analyses indicate that CG6255 is not expressed in $d y s^{E 6}$ but is expressed in $d y s^{G E 20705}$ at wild-type levels (supplemental Fig. 2, available at www.jneurosci.org as supplemental material).

We performed morphometric analyses of the muscle and motoneuron terminal at the well studied muscle 6/7 synapse (Atwood et al., 1993) to examine whether the dystrophin mutant NMJ or muscle displays any structural abnormalities. Wild-type and mutant body walls were labeled with anti-HRP, and muscle size, numbers of boutons, lengths of the synaptic termini, and the number of terminal branches were determined. No striking statistically significant differences were observed between dystrophin mutants and control larvae (supplemental Fig. 3, available at www.jneurosci.org as supplemental material). Furthermore, no apparent changes were seen in the distribution of the postsynaptically localized glutamate receptor subunits DGluRIIA and DGluRIIB, components of the major neurotransmitter receptors at the Drosophila NMJ (Fig. 3C-F). Because mammalian $d y$ strophin isoforms have been reported to be found associated with T-tubules (Watkins et al., 1988), invaginations of the sarcolemma containing voltage-gated channels, we examined the distribution of the Discs-large protein, which labels the T-tubuli (Razzaq et al., 2001), in the dystrophin mutant. Because Dystrophin colocalizes extrasynaptically with actin, we also examined actin localization in the mutant. Both proteins exhibit wild-type expression in $d y s^{E 6}$, suggesting that general muscle morphology is not affected in the dystrophin mutants (Fig. 3).

The lack of overt muscle degeneration in the dystrophin mutants allowed us to examine whether the large Dystrophin isoforms play a role in synaptic transmission. We measured the depolarizations following from spontaneous and evoked neurotransmitter release with an intracellular microelectrode at NMJs of muscle 6 in wild-type and dystrophin mutant female thirdinstar larvae in medium containing $1.5 \mathrm{mM} \mathrm{Ca}^{2+}$ (Fig. $4 A-E$ ). EJP amplitudes, evoked by nerve stimulation at $0.3 \mathrm{~Hz}$, were $\sim 45 \%$ increased $(p<0.05)$ in dystrophin mutants compared with the control larvae (Fig. $4 D$ ). Spontaneous mEJP amplitudes were essentially unchanged in the mutants compared with the controls (Fig. 4C). QC, the number of neurotransmitter quanta released on a nerve impulse, was calculated by dividing the mean EJP amplitude, corrected for nonlinear summation (Martin, 1955), by the mean mEJP amplitude. QC in the mutants, $d y s^{E 6}$ and $d y s^{G E 20705}$, was $\sim 50-65 \%$ higher $(p<0.05)$ than at wildtype control synapses (Fig. $4 E$ ). Calculation of QC without correction for nonlinear summation also resulted in statistically significant differences between the dystrophin mutants and wildtype controls (data not shown). In addition, the frequency of spontaneous neurotransmitter release was elevated $\sim 35-50 \%$ $(p<0.05)$ in the mutants (Fig. $4 B)$. These results indicate that postsynaptic dystrophin is required to maintain wild-type levels of neurotransmitter release.

In addition, the inter-allelic $d y s^{E 6} / d y s^{G E 20705}$ combination, the mutants each derived from an independent genetic background,
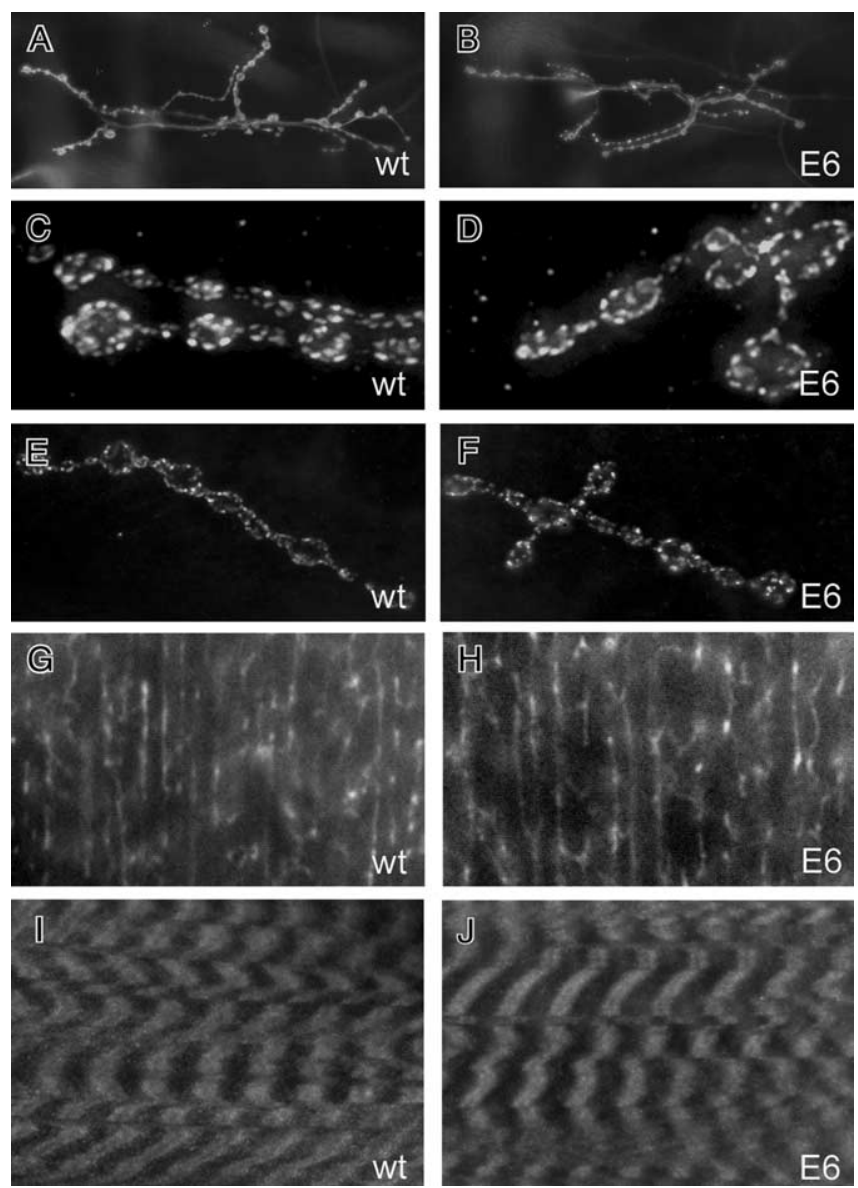

Figure 3. Synapse morphology and the distribution patterns of the DGluRIIA, DGluRIIB, Discs-large, and actin proteins are unchanged in the dystrophin mutants. Third-instar larval body walls were stained with anti-HRP, an antibody that labels all type I boutons $(\boldsymbol{A}, \boldsymbol{B})$, with anti-DGluRIIA (C, D), anti-DGluRIIB $(\boldsymbol{E}, \boldsymbol{F})$, anti-Discs-large $(\boldsymbol{G}, \boldsymbol{H})$, or anti-actin $(\boldsymbol{I}, \boldsymbol{J}) . \boldsymbol{A}, \boldsymbol{C}, \boldsymbol{E}, \boldsymbol{G}$, I, Representative wild-type (wt) muscle 6/7 NMJs. $\boldsymbol{B}, \boldsymbol{D}, \boldsymbol{F}, \boldsymbol{H}, \boldsymbol{J}$, Representative dys ${ }^{E 6}$ (E6) muscle $6 / 7$ NMJs. No significant changes in the distribution pattern of the examined proteins are observed in the dystrophin mutant compared with the wild-type control larvae.

showed a similar effect on EJPs, mEJPs, QC, and mEJP frequency as the homozygous mutants, further indicating that the phenotype observed was attributable to the mutation in dystrophin (Fig. 4). Interestingly, we saw a similar electrophysiological behavior in the heterozygotes, $d y s^{E 6} /+$ and $d y s^{G E 20705} /+$ (Fig. 4), indicating that dystrophin is haplo-insufficient for normal Drosophila NMJ physiology. Semiquantitative RT-PCR analysis shows that the DLP2 levels are reduced twofold to fourfold in the heterozygote animals (supplemental Fig. 2, available at www. jneurosci.org as supplemental material).

To determine whether the elevated neurotransmitter release in the mutant showed altered $\mathrm{Ca}^{2+}$ dependence, we compared the QCs of dystrophin mutant NMJs with controls measured at external $\mathrm{Ca}^{2+}$ concentrations ranging from 0.25 to $1.5 \mathrm{mM}$. $\mathrm{Mu}-$ tant NMJ QC was significantly higher than the controls at all concentrations tested (Fig. 5A). The slopes of the regression lines of the $\mathrm{Ca}^{2+}$ titration curves at the low $\mathrm{Ca}^{2+}$ range (0.25-0.6 mM) did not differ significantly, showing that the $\mathrm{Ca}^{2+}$ cooperativity at the mutant NMJ is apparently unchanged. Thus, the increased release observed in the dystrophin mutant is independent of the extracellular $\mathrm{Ca}^{2+}$ concentration and unlikely to be attributable to altered properties of the $\mathrm{Ca}^{2+}$ sensor that triggers presynaptic vesicle fusion. 
We wanted to confirm the increase in QC using calculations independent of the mEJP amplitudes. Therefore, we applied failure analysis and the variance method (Boyd and Martin, 1956; Martin, 1966). Both failure and variance analyses performed on EJP data obtained at $0.25 \mathrm{~mm}$ $\mathrm{Ca}^{2+}$ demonstrated a similar increase in QC as calculated using the direct method (Fig. 5B). These results indicate that the increase in EJP amplitude is attributable to an increase in presynaptic glutamate release in the mutants.

Elevated QC can result from increased probability of release or increases in the size of the readily releasable vesicle pool. Because increased probability has been shown to be reflected by reduced pairedpulse facilitation (Rohrbough et al., 1999; Sandstrom, 2004), we determined this short-term dynamic behavior of neurotransmitter release in wild-type and $d y s^{E \sigma}$ larvae (Fig. 5C). The protocol resulted in $\sim 50 \%$ facilitation in wild-type larvae but failed to do so in the $d y s^{E 6}$ mutant $(p<$ $0.05)$. The size of the readily releasable vesicle pool can be assessed experimentally by measuring asynchronous quantal release during incubation with hypertonic medium (Stevens and Tsujimoto, 1995). Application of $0.05 \mathrm{M}$ sucrose did not reveal significant differences in the elevated mEJP frequency level between the mutant and wild type (Fig. 5D). These analyses indicate that the elevation in evoked neurotransmitter release at the dystrophindeficient NMJ resulted from increased probability of release rather than from an increased size of transmitter vesicle pool.

\section{Transgenic RNA interference and rescue experiments show a postsynaptic requirement for Dystrophin}

Our RT-PCR analyses indicated that the $d y s^{E 6}$ mutation reduces the expression levels of DLP2 and CG6255 and $d y s^{G E 20705}$ affects DLP2 and the CNS-specific isoform Dp186 but not CG6255. To further address potential roles for either CG6255 or Dp186 in the dystrophin mutant NMJ phenotype and to confirm that the postsynaptic absence of Dystrophin protein is responsible for the increased neurotransmitter release in the mutants, we used transgenic tissue-specific RNA interference to reduce Dystrophin levels specifically either presynaptically or postsynaptically. We generated transgenic flies that express double-stranded RNA (dsRNA) directed against dystrophin sequences present in the large $d y$ strophin isoform mRNAs (UAS-RNAi-dys $\mathrm{NH}_{2}$ ) under Gal4 control (Brand and Perrimon, 1993). Semiquantitative RT-PCR analysis shows a more than eightfold decrease of DLP2 expression levels in the larval musculature during expression of UAS-RNAidysNH2 driven by 24B-Gal4, but Dp186 and CG6255 levels remained at the levels observed in both wild-type and the $d y s^{E 31}$ precise excision larvae (supplemental Fig. 2, available at www. jneurosci.org as supplemental material). Pan-neuronal expres-
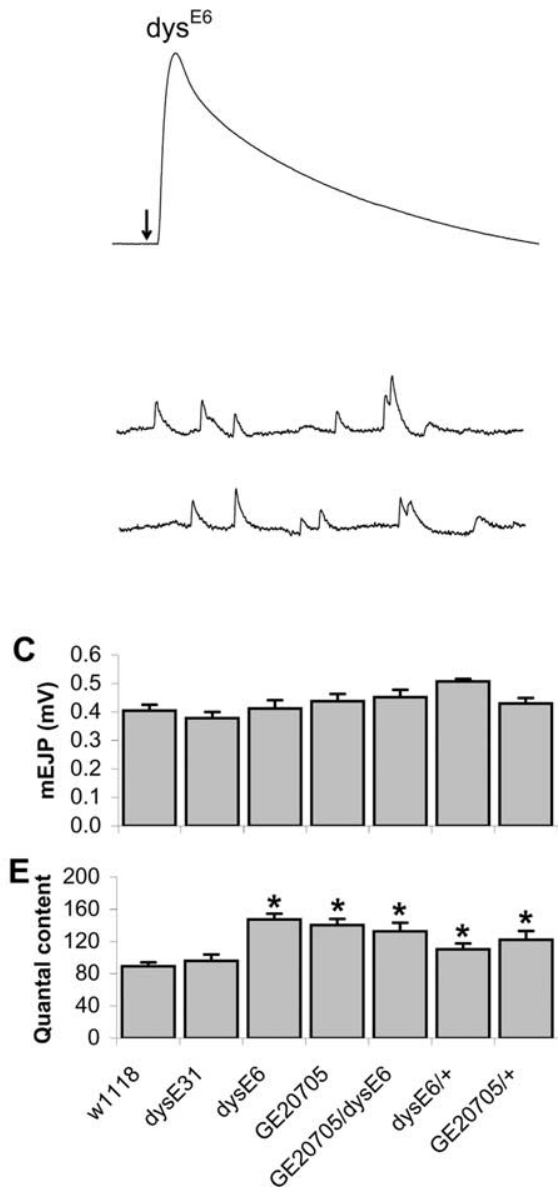

Figure 4. Electrophysiological analysis of dystrophin mutants reveals a role for dystrophin in regulating quantal content at the arrow indicates the moment of stimulation (stimulation artifact removed for clarity). $\boldsymbol{B}-\boldsymbol{E}$, Bar graph representations of mean \pm SEM values of mEJP frequency $(\boldsymbol{B})$, mEJP amplitude $(\boldsymbol{C})$, EJP amplitude $(\boldsymbol{D})$, and $Q \boldsymbol{C}(\boldsymbol{E})$ for the genotype $w^{1118}(n=11)$, the precise dys $^{G E 20705} /$ dys $^{E 6}(n=7)$, the dys $s^{E 6} /+$ heterozygote mutant $(n=5)$, and the dys ${ }^{G E 20705} /+$ heterozygote mutant $(n=3)$. All 列 $1.5 \mathrm{~mm} \mathrm{Ca}{ }^{2+}$. EJPs and QC are significantly increased in all dystrophin mutants, including with the controls. The frequency of spontaneous neurotransmitter release was elevated in the dys ${ }^{E 6}$ mutant [EJPs, $39.7 \pm 0.6 \mathrm{mV}$

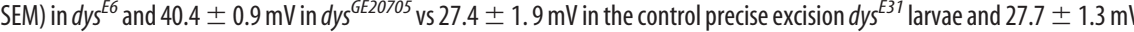
in the wild-type $\left(w^{1118}\right)$ larvae; QCs, $147.4 \pm 7.2$ in $d y s^{E 6}$ and $140.3 \pm 7.7$ in dys ${ }^{G E 20705}$ vs $95.9 \pm 8.0$ in the control precise excision dys ${ }^{E 31}$ larvae and $89.3 \pm 4.7$ in the wild-type larvae; $m E J P$ frequencies, $3.6 \pm 0.3 /$ in dys ${ }^{E 6}$ and $3.3 \pm 0.2 /$ s in dys ${ }^{G E 20705}$ vs $2.4 \pm 0.4 / \mathrm{s}$ in the control precise excision dys ${ }^{E 31}$ larvae and $2.4 \pm 0.2 / \mathrm{s}$ in the wild-type larvae]. ${ }^{*} p<0.05$.

sion of UAS-RNAi-dysNH $\mathrm{N}_{2}$, driven by Elav-Gal4, failed to suppress Dp186 expression levels, further confirming the specificity of the effects of this transgene. When UAS-RNAi-dys $\mathrm{NH}_{2}$ was expressed in the muscle using either of two different musclespecific drivers, 24B-Gal4 or G14-Gal4, QC increased to similar levels ( $\sim 70 \%$ higher than control) as observed in the $d y$ strophin mutants (Fig. 6). However, when this construct is driven by the Elav-Gal4 transgene, no change in QC compared with the control larvae was observed.

We then asked whether we could rescue the dystrophin mutant phenotype by increasing postsynaptic dystrophin expression. Lacking a rescue transgene, we took advantage of a P-element insertion, GS12472 (Toba et al., 1999), which lies $1.9 \mathrm{~kb}$ upstream of the DLP2 initiator codon and bears a UASdependent promoter appropriately oriented to express DLP2. Driving this P-element does not overexpress DLP1 or CG6255; the P-element insertion site is downstream of the unique first exon of DLP1, and CG6255 has the opposite orientation to the 
A

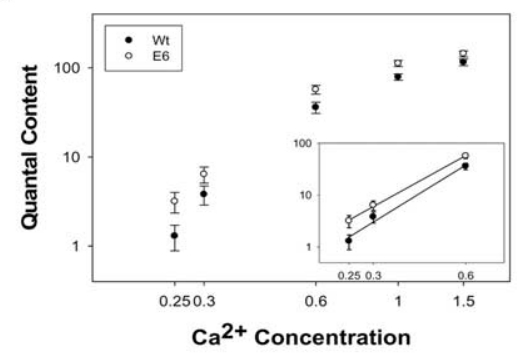

C

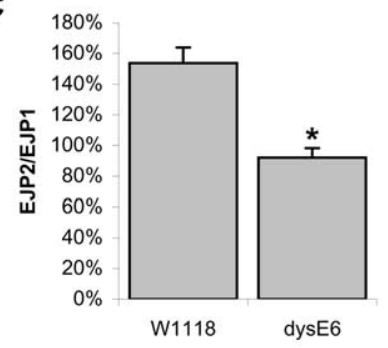

D

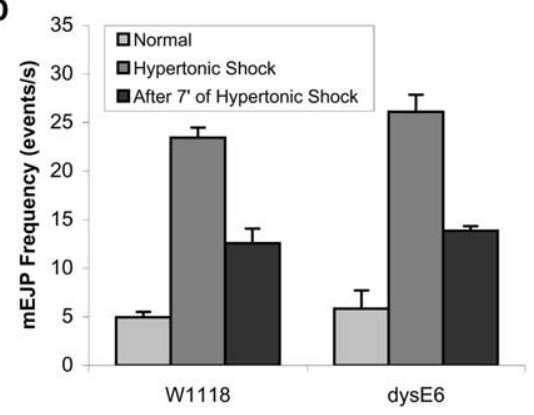

Figure 5. Quantal content over a range of $\mathrm{Ca}^{2+}$ concentrations and the probability of release are increased in dystrophin mutants. $A$, Double-logarithmic plot of the $Q C$ as a function of extracellular $\mathrm{Ca}^{2+}$ concentration for $w^{1118}$ and $d y s^{E 6}$. Data points represent the mean \pm SEM. QC was calculated from recordings from at least nine muscles for each $\mathrm{Ca}^{2+}$ concentration. The graph in the inset shows that there is no significant change in $\mathrm{Ca}^{2+}$ dependency in dys ${ }^{E 6}$ mutants (slope of $2.5 ; r^{2}=0.999$ ) compared with the wild type (wt) (slope of $\left.2.4 ; r^{2}=0.985\right)$. $B$, Besides using the direct method, the QC was calculated by application of the failure analysis and the variance method. Both methods confirmed the increase of the QC in the dys ${ }^{E 6}$ compared with the wild-type larvae at $0.25 \mathrm{~mm} \mathrm{Ca}^{2+}\left(\mathrm{QCs}\right.$ for $w^{1118}$ controls vs dys $^{E 6}$, direct method, $1.4 \pm 0.3 \mathrm{vs} 3.5 \pm 0.6$; failure analysis, $0.9 \pm 0.1$ vs $2.8 \pm$ 0.4 ; and variance method, $1.3 \pm 0.2$ vs $4.3 \pm 0.7) .{ }^{*} p<0.05$. C, Paired-pulse facilitation of EJP amplitude during two stimuli delivered with a $50 \mathrm{~ms}$ interstimulus interval measured at $0.6 \mathrm{~mm} \mathrm{Ca}{ }^{2+}$ is shown in the graph as EJP2/EJP1, with the EJP1 normalized to $100 \%$. The traces are representative responses of wild-type and $d y s^{E 6}$ larval NMJs at 50 ms interstimulus interval. In contrast to the wild type ( $n=7$ larvae), facilitation does not occur in the dys ${ }^{E 6}$ larvae ( $n=5$ larvae), indicating an increase in probability of release of synaptic vesicles ( $w^{1118}$ EJP2/EJP1, $153.8 \pm 10.2 \%$; dys ${ }^{E 6}$ EJP2/EJP1, $92.0 \pm 6.1 \%$ ). ${ }^{*} p<0.05 . D$, Wild-type (15 NMJs of 5 larvae) and $d y s^{E 6}$ (12 NMJs of 4 larvae) larvae show the same elevated mEJP frequency level during incubation with $\mathrm{HL} 3$ containing $50 \mathrm{~mm}$ sucrose (hypertonic shock), indicating that the readily releasable pool of synaptic vesicles is unchanged in the $d y s^{E 6}$ mutant ( $w^{1118} \mathrm{mEJP}$ frequency before addition of sucrose, $5.0 \pm 0.6 / \mathrm{s}$; during, $23.4 \pm 1.9 / \mathrm{s}$; and $7 \mathrm{~min}$ after, $12.6 \pm 1.5 / \mathrm{s} ; \mathrm{dys}^{E 6} \mathrm{mEJP}$ frequency before addition of sucrose, $5.8 \pm 1.0 / \mathrm{s}$; during, $26.1 \pm 1.8 / \mathrm{s}$; and $7 \mathrm{~min}$ after, $13.8 \pm$ $0.5 / 5)$.

dystrophin coding frames. Overexpression of DLP2 was confirmed by Western blot analysis of transgenic embryos (Fig. $2 I$ ). Postsynaptic expression of the DLP2 isoform in the heterozygous mutant background rescues QC to wild-type levels (G14-Gal4/+; GS12472/dys ${ }^{E 6}$ ) but not when expressed presynaptically (OK6-Gal4/+; GS12472/dys ${ }^{E 6}$ ) (Fig. 6). Together with the mutant analyses, these data indicate that electrophysiological phenotype at the $d y s^{E 6}$ and $d y s^{G E 20705}$ mutant NMJs is elicited by decreased expression of the postsynaptically expressed DLP2 isoform and is unlikely to be attributable to effects during CG6255, Dp186, or presynaptically expressed DLP2.
The absence of postsynaptic Dystrophin results in an increase in the number of T-bars

To evaluate whether the electrophysiological changes in the mutant correlate with alterations in NMJ ultrastructure, we performed morphometric measurements on electron micrographs of mutant and control third-instar larval NMJs (Fig. 7) (supplemental Fig. 4, available at www. jneurosci.org as supplemental material). The bouton area and the surface area of the subsynaptic reticulum relative to the bouton area were similar for all genotypes, but the shape of the boutons in the mutants $d y s^{G E 20705}$ and $d y s^{E 6}$ appeared slightly more elongated, as determined by the ratio of the longest and shortest diameter of a bouton section (supplemental Fig. 4, available at www.jneurosci.org as supplemental material). The area of the bouton occupied by vesicles was increased in the $d y s^{G E 20705}$ $(85.2 \pm 1.6 \% ; p<0.05)$ and $d y s^{E G}(80.5 \pm$ $2.6 \%)$ mutants compared with wild type $(73.2 \pm 3.2 \%)$ and precise excision control $d y s^{E 31}(74.4 \pm 2.8 \%)$. T-bars are electrondense presynaptic structures possibly representing a subtype of neurotransmitter release site (Jia et al., 1993). The number of active zones with a T-bar relative to the total number of active zones was significantly increased in both the $d y s^{E G}$ and $d y s^{G E 20705}$ mutants by approximately twofold $\left(d y s^{E G}, 0.61 \pm 0.05 ; d y s^{G E 20705}, 0.74 \pm\right.$ 0.05 ; wild type, $0.39 \pm 0.03 ; p<0.05$ ) (Fig. 7 ), whereas the overall number of active zones did not increase. Although wildtype T-bar densities vary considerably between laboratories and their precise function is unclear, we report this increase in T-bars in the mutant relative to the isogenic control as an observed ultrastructural correlate of the increased neurotransmitter release in the dystrophin mutant.

\section{BMP signaling at the NMJ is required for the increased neurotransmitter release in the Dystrophin mutant}

The BMP pathway plays an important role in retrograde signaling at the larval NMJ (for review, see Keshishian and Kim, 2004) and in the Drosophila CNS (Baines, 2004). To evaluate the role of BMP signaling in the increase in neurotransmitter release induced by the absence of dystrophin, we performed electrophysiological recordings at NMJs lacking both dystrophin and wit (Fig. 8). We used the transgenic RNA interference approach described above to reduce postsynaptic dystrophin levels.

Homozygous wit mutant NMJs postsynaptically expressing dsRNA directed against the Dystrophin large isoforms (UASRNAi-dysNH2/+; G14-Gal4/+; wit) displayed mEJP amplitude, EJP amplitude, and QC values similar to the wit mutant alone. As shown previously (Marques et al., 2002), the homozygous wit 
NMJ has very low EJP amplitudes (Fig. $8 D$ ) and low QC (Fig. $8 E$ ) but maintains wild-type level mEJP amplitudes. Thus, the absence of $d y$ strophin failed to elicit an increase in QC in the wit background. Wit function and, by extension, BMP signaling is therefore required at the NMJ for the increase in neurotransmitter release elicited by the absence of dystrophin.

To evaluate whether the absence of postsynaptic dystrophin affects a known BMP target, we performed immunofluorescence analysis of mutant and wild-type embryonic ventral nerve cords, using an anti-phospho-Mad (PMad) antibody that recognizes the activated form of the Mad downstream effector of wit signaling (Tanimoto et al., 2000; Marques et al., 2002). We did not observe any differences in PMad expression levels or domains between the dystrophin mutants or individuals postsynaptically overexpressing DLP2 and controls, whereas homozygous wit mutants showed significantly decreased levels of the protein, as reported previously (supplemental Fig. 5, available at www. jneurosci.org as supplemental material) (Marques et al., 2002).

The dystrophin mutants show similar electrophysiological and morphological phenotypes as larvae postsynaptically expressing CaMKII inhibitors (Haghighi et al., 2003). Both display increased QC and an increase in the ratio of active zones with T-bars versus the total number of active zones, without additional significant changes in synaptic morphology. Furthermore, the increase in QC when CaMKII is reduced postsynaptically is dependent on wit function. Therefore, we examined embryonic ventral nerve cords that have reduced or elevated levels of CaMKII, UASAla, and UAS-T287D, respectively, for PMad staining. No changes in the expression of PMad were observed (supplemental Fig. 5, available at www.jneurosci.org as supplemental material). These results suggest that, although wit is required for increased QC at NMJs postsynaptically deficient for either Dystrophin or CaMKII, alteration of either Dystrophin or CaMKII levels does not result in changes in embryonic PMad expression. Thus, the interaction of dystrophin or CaMKII and wit in NMJ homeostasis is unlikely to involve regulation of PMad expression.

\section{Discussion}

This study provides evidence that postsynaptic dystrophin is required to maintain appropriate levels of presynaptic neurotransmitter release. The absence of dystrophin from the muscle results in abnor-
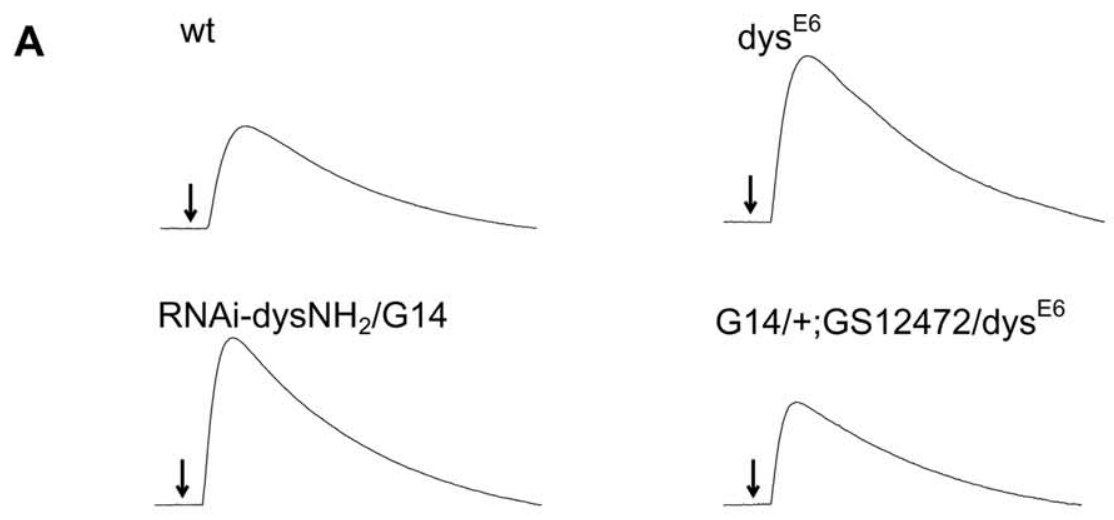

G14/+;GS12472/dys ${ }^{\mathrm{E} 6}$
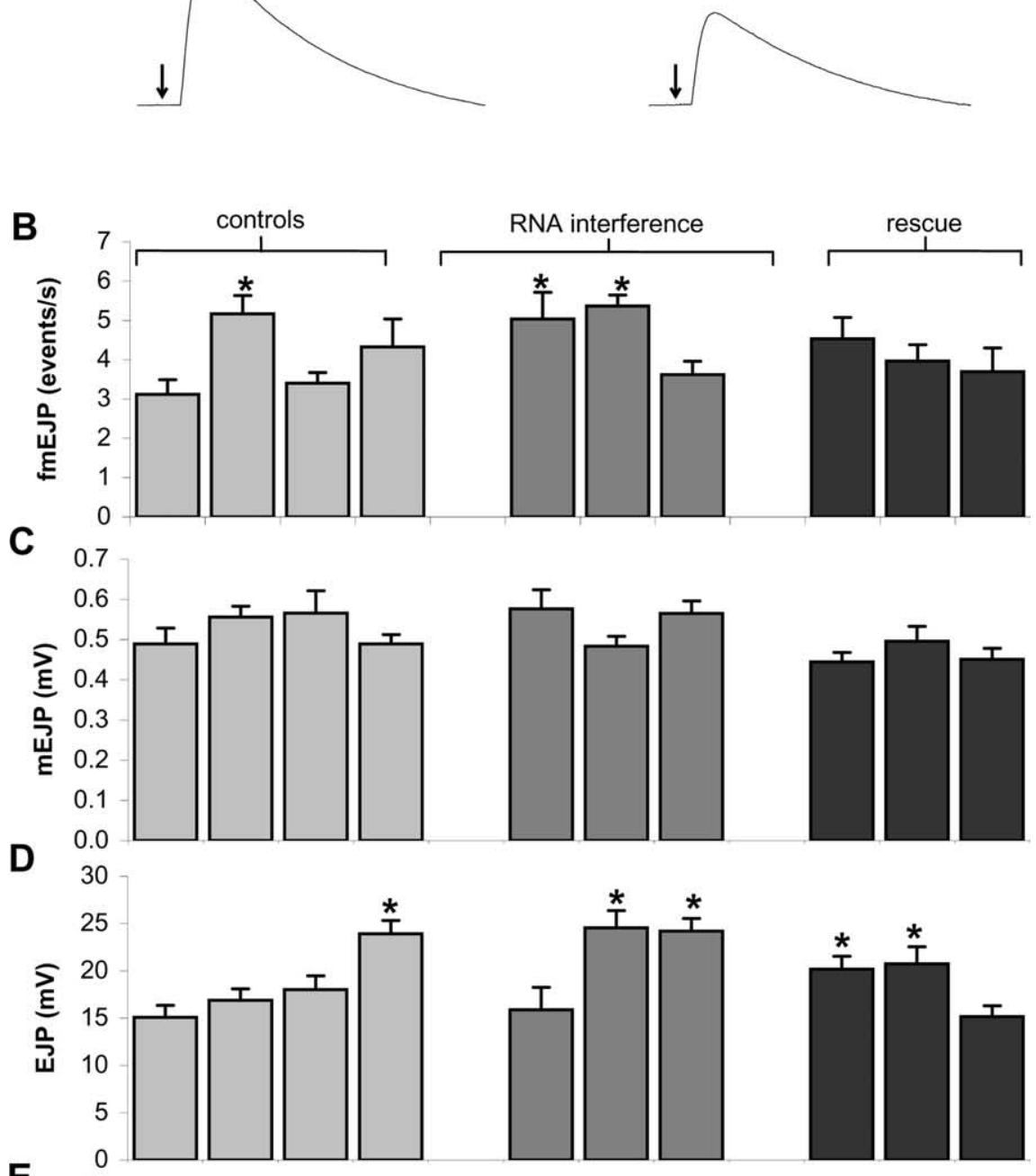

$\mathbf{E}$

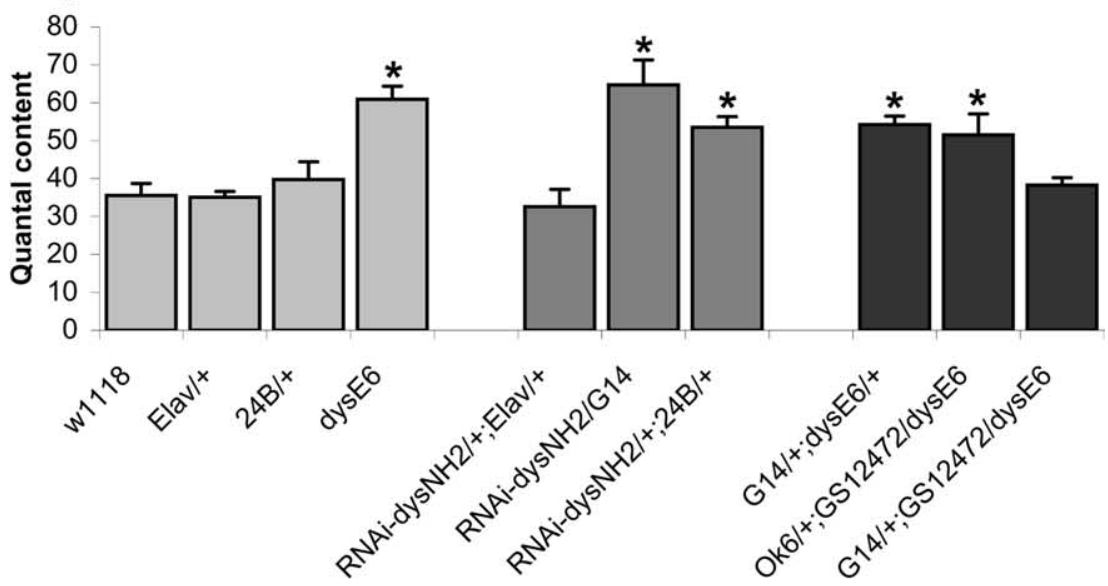

Figure 6. RNA interference and rescue experiments show a postsynaptic requirement for the large Dystrophin isoforms at the larval NMJ. $\boldsymbol{A}$, Representative traces of EJPs recorded from NMJs of wild-type (wt; $w^{1718}$ ) control, the mutant $d y s^{E 6}$, and the genotypes RNAi-dys $\mathrm{NH}_{2} / \mathrm{G} 14-\mathrm{Gal} 4$ in which the large Dystrophin isoforms are downregulated and (Figure legend continues.) 


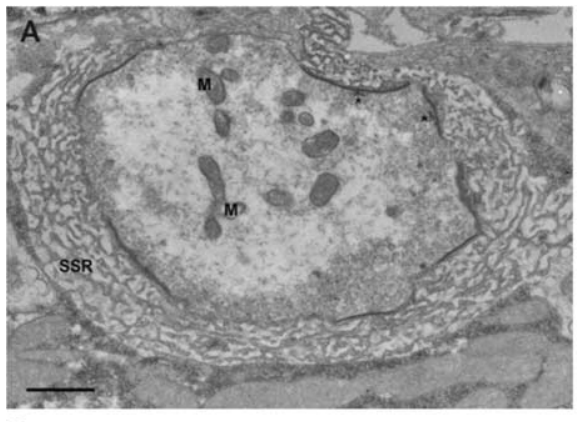

C

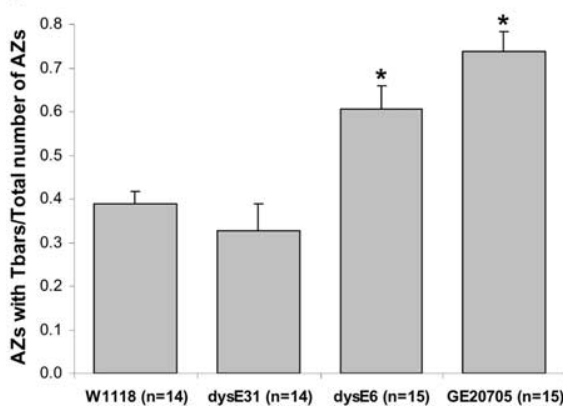

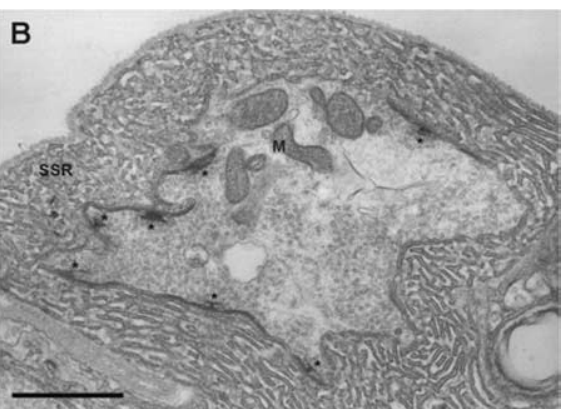

D

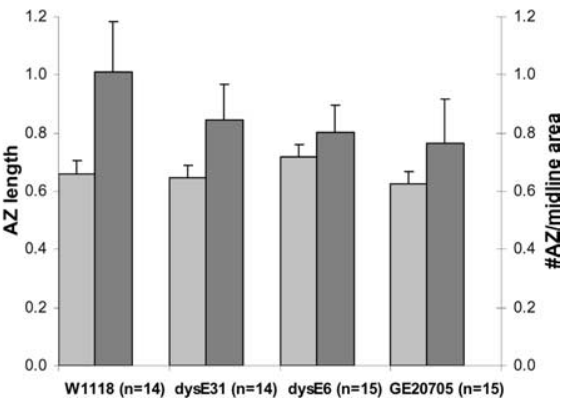

Figure 7. The number of T-bars is increased in dystrophin mutants. Third-instar larval body walls were prepared by ultrathin sectioning, and the muscle 6 and 7 synapses were visualized by electron microscopy. $\boldsymbol{A}, \mathrm{A}$ representative type $\mathrm{lb}$ bouton in a wild-type larva. $\boldsymbol{B}$, A representative type lb bouton in a $d y s^{E 6}$ mutant larva. T-bars are indicated by asterisks; $M$, mitochondria; SSR, subsynaptic reticulum. Scale bars, $1 \mu \mathrm{m}$. $\boldsymbol{C}$, The graph shows the mean of the number of active zones with T-bars relative to the total number of active zones, based on the quantitative analysis of $84,71,107$, and 58 active zones in at least 14 individual bouton sections at the midline for $w^{1118}$, dys $s^{E 31}$, dys ${ }^{E 6}$, and dys ${ }^{G E 20705}$ ( $n$ value). A significant increase in T-bars is observed in the mutants, $d y s^{E 6}$ and $d y s^{G E 20705}$, but not in the precise excision control dys $s^{E 31}$ relative to wild-type larvae. ${ }^{*} p<0.05$. D, The graph shows that neither the average length of active zones at the midline is significantly different between the genotypes nor the number of active zones normalized to the midline area of the bouton. The averaged numbers of active zones per bouton midline section are as follows: six for $w^{1118}$, five for $d y s^{E 31}$, seven for $d y s^{E 6}$, and five for $d y s^{G E 20705}$. AZ indicates active zones.

mally high QC as determined by three different methods. The NMJ homeostatic machinery is apparently engaged in the absence of dystrophin, but it has an inappropriate endpoint. In contrast to members of the BMP signaling pathway implicated previously in NMJ homeostasis (Aberle et al., 2002; Marques et al., 2002; McCabe et al., 2003) but similarly to CaMKII (Haghighi et al., 2003), Dystrophin does not appear to regulate synaptic growth or overall structure. The requirement for dystrophin is haplo-insufficient, indicating that the Drosophila NMJ is highly sensitive to the levels of Dystrophin, possibly reflecting its interaction with rate-limiting signaling factors.

We show in a number of experiments that the lack of the postsynaptically localized DLP2 isoform is responsible for the electrophysiological phenotype observed in the dystrophin mutants: (1) expression analyses and semiquantitative RT-PCR

(Figurelegend continued.) G14-Gal4/+;GS12472/dys ${ }^{E G}$ in which the large isoform DLP2 is overexpressed in the muscle in the dys ${ }^{E G}$ mutant background. $\boldsymbol{B}-\boldsymbol{E}$, Bar graph representations of mean \pm SEM values of $m E$ EJP frequency $(\boldsymbol{B}), \mathrm{mEJP}$ amplitude $(\boldsymbol{C})$, EJP amplitude $(\boldsymbol{D})$, and $Q C(\boldsymbol{E})$ for the control genotypes [wild type $\left(w^{1118}\right)(n=5)$, Elav-Gal4/ $+(n=6), 24 \mathrm{~B}-\mathrm{Gal} 4 /+(n=8)$, and $\left.\operatorname{dys}^{E 6}(n=8)\right]$, for the transgenic RNA interference genotypes, large-isoform-specific RNAi-dysNH $2 /+$; Elav-Gal $4 /+(n=7)$, RNAi-dysNH $2 /$ G14-Gal4 $(n=8)$, RNAi-dysNH $/ 2+; 24 \mathrm{~B}-\mathrm{Gal} 4 /+(n=7)$, and, for the rescue experiment, G14-Gal4/+; dys ${ }^{E 6} /+$

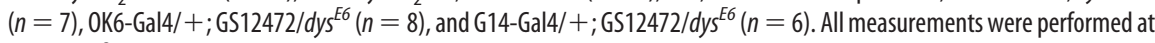
$0.6 \mathrm{~mm} \mathrm{Ca}^{2+}$.QC is significantly increased by postsynaptic expression of dsRNAs directed against the large dystrophin isoforms (RNAi-dysNH $2 /+; 24 B-G a 14 /+$ or RNAi-dysNH $2 /$ G14-Gal4) but not when the large-isoform-specific dsRNA is expressed only in the motoneuron (RNAi-dysNH $/ 2 /+$; Elav-Gal4/+). Expression of the DLP2 isoform in the mutant background reduces QC to wild-type levels when it is expressed postsynaptically (G14-Gal4/+; GS12472/dys ${ }^{E 6}$ ) but not presynaptically (OK6-Gal4/+;

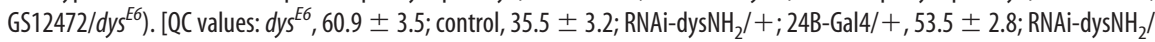
G14-Gal4, 64.7 \pm 6.6; RNAi-dysNH $/ 2$; Elav-Gal4/+, $32.6 \pm$ 4.6; G14-Gal4/+; GS12472/dys ${ }^{E 6}, 38.3 \pm 1.9 ; 0 K 6-G a l 4 /+$;

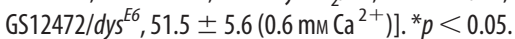

studies indicate that, of the known dystrophin isoforms, only expression of DLP2 is affected by the mutation in $d y s^{E G}$, (2) the mutant phenotype can be rescued by expressing DLP2 postsynaptically but not presynaptically, (3) expression of doublestranded RNA directed against sequences present in the DLP2 isoform results in increases in neurotransmitter release similar to those observed in the $d y s^{E 6}$ mutants, only when expressed postsynaptically, (4) RNA in situ analyses and RT-PCR assays show that DLP2 is highly expressed in larval muscle, DLP1 is not detectably expressed in the musculature, and all dystrophin large isoforms are absent from the neuropil in which the cell bodies of motoneurons are located, and (5) double labeling of larval body walls with antiDystrophin and anti-HRP, which labels the presynaptic membrane, reveals no overlapping expression domains.

We performed morphometric analyses and stained with several muscle- and motoneuron-specific antibodies to examine the general morphological characteristics of the synapse and musculature in the mutants. These studies indicate that muscle and synapse size are not significantly altered by the lack of Dystrophin. Because we found that Dystrophin colocalizes extrasynaptically with actin, we examined actin distribution in the mutant muscle but did not observe differences relative to the controls. The morphology of the T-tubular network was also unaltered. That the absence of dystrophin does not grossly affect muscle morphology or glutamate receptor density/properties was reflected by our observation of unchanged electrical input resistance, $\mathrm{mEJP}$ amplitudes, and anti-glutamate receptor subnit antibody staining in the mutants.

Although DLP2 is expressed only postsynaptically and changes in synapse elaboration or muscle morphology were not observed in its absence, there are significant changes in the NMJ electrophysiology of the mutant. EJP amplitudes are increased with little or no change in the mEJPs, resulting in increased QC, i.e., the number of quanta released on stimulation, calculated by the direct, failure, or variance methods. We therefore examined presynaptic mechanisms whose alteration might account for the increase in QC at the mutant NMJ. The increase was correlated to an increase in the probability of release as indicated by reduced EJP facilitation at the mutant synapse (Schulz et al., 1994; Sandstrom, 2004).

In addition to increased release probability, an increased size of the readily releasable vesicle pool may account for the increased QC at the dystrophin mutant NMJ. However, this seems not the case in the dystrophin mutant because the increase in $\mathrm{mEJP}$ frequency at the mutant $\mathrm{NMJ}$ in response to hypertonic shock, a method used to assess the readily releasable pool (Stevens and Tsujimoto, 1995; Suzuki et 
al., 2002), did not differ from that at control NMJs. It should, however, be noted that it is not yet clear that only the readily releasable pool of vesicles is released after hypertonic shock of the Drosophila larval NMJ (Kidokoro et al., 2004).

Increased release probability may result from increased efficacy of the translation of presynaptic $\mathrm{Ca}^{2+}$ influx into transmitter release by the neuroexocytotic machinery, possibly attributable to increased sensitivity of $\mathrm{Ca}^{2+}$ sensors. However, our observation that both the mutant and wild-type NMJs have highly similar slope values for the $\log \left[\mathrm{Ca}^{2+}\right]$ versus $\log [\mathrm{QC}]$ argues against such an effect. An alternative explanation is that aberrant retrograde signaling in the absence of dystrophin results in altered modulation of presynaptic $\mathrm{Ca}^{2+}$ channel activity and thus leads to increased presynaptic $\mathrm{Ca}^{2+}$ influx.

Ultrastructural analysis of synaptic boutons showed a slight increase of the area occupied by vesicles in the mutants, which is unlikely to be sufficient to explain the increase in QC. Also, we did not observe significant changes in the number and size of active zones, whose increase could explain the increased neurotransmitter release. We did observe an increase in T-bars, the presumed docking sites for synaptic vesicles, in the mutant boutons. Although at present the precise function of these morphologically defined structures is unclear, an increase in T-bars has also found correlated with increased neurotransmitter release in other studies (Haghighi et al., 2003).

How does the absence of dystrophin affect the retrograde control of release? The abnormally high EJP amplitudes observed at the dystrophin mutant NMJ suggests the possibility that the Dystrophin-deficient muscle inappropriately signals to the motoneuron that it is inadequately depolarized. Thus, the absence of dystrophin may result in desensitization of an as yet unknown monitor of muscle function during depolarization. This raises the following questions: what is the homeostatic monitor and how does Dystrophin interact with it? Although a postsynaptic monitor regulating NMJ homeostatic pathways has been proposed (Davis and Bezprozvanny, 2001), its identity has proven elusive. The ligand-gated glutamate receptor channel, which conducts $\mathrm{Ca}^{2+}$, has been an attractive candidate (Petersen et al., 1997; Davis et al., 1998; DiAntonio et al., 1999), and mammalian Dystrophin is known to scaffold a variety of postsynaptic $\mathrm{Ca}^{2+}$ channels (for review, see Carlson, 1998). However, we do not observe alterations in either glutamate receptor field sensitivity to spontaneous neurotransmitter release or localization of the DGluRIIA or DGluRIIB subunits in the dystrophin mutant, suggesting that glutamate receptors are unlikely to play a key role in the homeostatic pathways affected by the lack of dystrophin.

Glutamate receptor-independent monitors of muscle depolarization, possibly voltage-gated L-type channels, have been shown sufficient to trigger NMJ homeostatic mechanisms (Para-
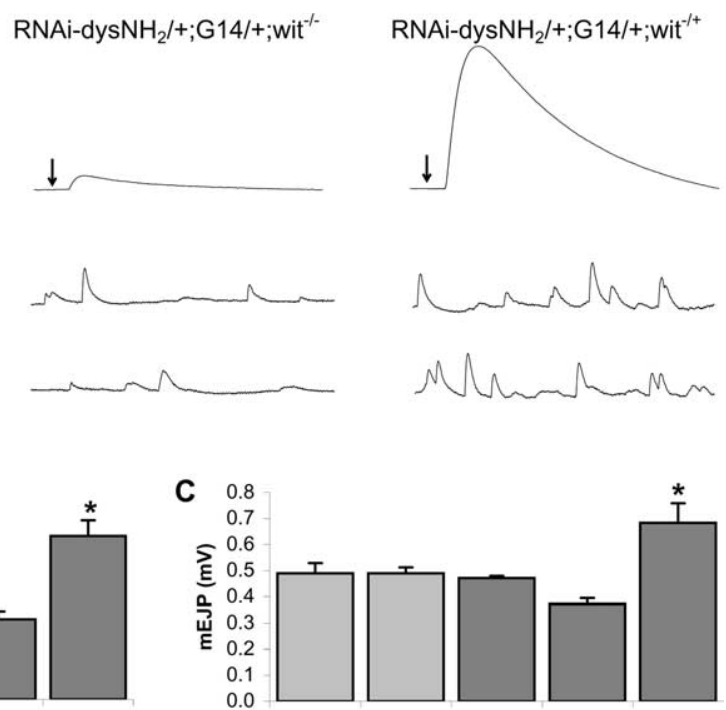

E 70
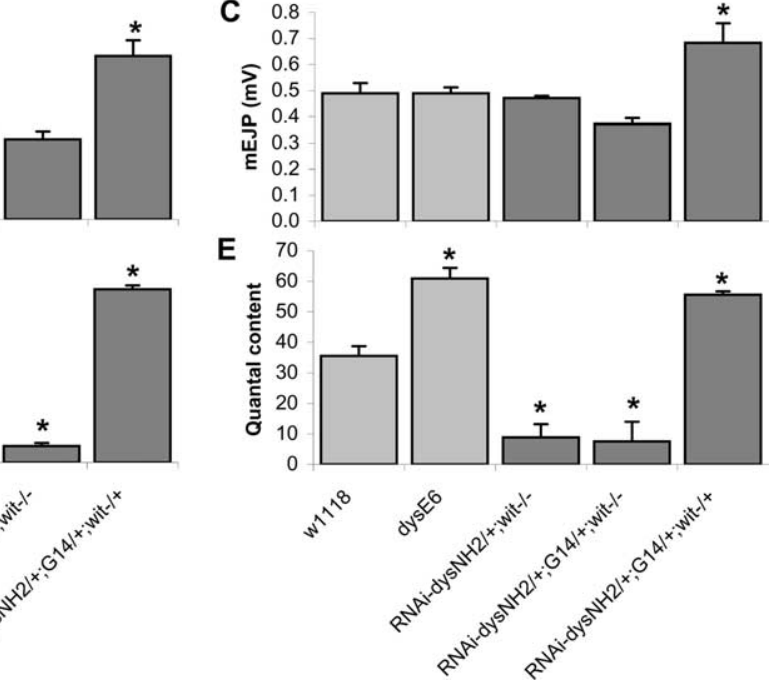

Figure 8. Increases in quantal content in the absence of dystrophin require the wit BMP receptor. $A$, Representative traces of EJPs and mEJPs recorded at NMJs from wild-type (wt; $w^{1118}$ ) control and the genotypes RNAi-dysNH2/+;G14-Gal4/+; wit ${ }^{A 2}$ / ${ }_{i}^{B 11}$ and RNAi-dysNH2/+;G14-Gal4/+; wit ${ }^{A 12} /+$. B $-E$, Bar graph representations of mean \pm SEM values of mEJP frequency

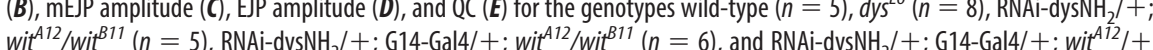
amplitude and QC (EJP values, $3.9 \pm 0.7 \mathrm{mV}$ in the wit mutant vs $15.1 \pm 1.3 \mathrm{mV}$ in wild type; $\mathrm{QC}$ values, $8.9 \pm 1.1$ in the wit mutant vs $35.5 \pm 3.2$ in wild type, $\left.0.6 \mathrm{~mm} \mathrm{Ca}^{2+}\right)$. The UAS-RNAi-dysNH2/+;G14-Gal4/+; wit NMJs displayed mEJP, EJP, and QC values similar to the wit mutant. ${ }^{*} p<0.05$.

dis et al., 2001). Alteration of L-type $\mathrm{Ca}^{2+}$ channel function or other changes in $\mathrm{Ca}^{2+}$ handling in the dystrophin-deficient larval muscle, which are well documented in mammals (for review, see Gailly, 2002), might inappropriately trigger or prolong the action of the homeostatic machinery, resulting in aberrantly high neurotransmitter release and muscle hyperdepolarization. CaMKII and $\mathrm{Ca}^{2+}$ /calmodulin represent other attractive candidates to directly link Dystrophin to homeostatic pathways; $\mathrm{Ca}^{2+} /$ calmodulin has been shown to associate with the DGC (Madhavan et al., 1992), but its role in dystrophin function is not yet understood. Possibly, dystrophin indirectly affects cAMP-dependent mechanisms (Johnson et al., 2005) that have been shown to be involved in synaptic plasticity and short-term dynamics of release (Zucker and Regehr, 2002).

We find that the presynaptically localized type II BMP receptor wit is required for the increased QC observed in the dystrophin mutant, as also shown for increases in QC induced by the postsynaptic inhibition of CaMKII or glutamate receptor function (Haghighi et al., 2003). Dystrophin and CaMKII are unlikely, however, to signal through the PMad-dependent BMP signaling pathway, because we find that the expression levels and domains of PMad are unchanged when Dystrophin or CaMKII 
levels are either decreased or increased. Our results address the previously posed question (Sanyal et al., 2004) as to whether the retrograde BMP signal directly participates in homeostatic signaling ("instructive") or is required for the overall development of the synapse ("permissive"). Our findings indicate that it is likely permissive, at least for the homeostatic mechanisms induced by perturbation of Dystrophin or CaMKII levels. BMP signaling may simply be required for the development of the presynaptic apparatus to a point at which it can respond to muscle-derived cues.

The degree of evolutionary conservation of the role of $d y$ strophin in regulating neurotransmitter release at the NMJ and the potential role that defective NMJ homeostasis may play in the onset or progression of muscular dystrophy in humans is, at present, unclear. Differences between species in the severity of muscle wasting in the absence of dystrophin are observed (Megeney et al., 1996; Segalat, 2002). We do not observe muscle degeneration when DLP2 is absent from the musculature; other isoforms may be required for muscle integrity in Drosophila. The dystrophin-deficient cholinergic Caenorhabditis elegans NMJ also shows elevated levels of neurotransmitter, but this is attributable to decreased clearance of acetylcholine subsequent to delocalization of the SNF-6 acetylcholine transporter (Kim et al., 2004). Recent work on retinal synapses in the $m d x$ mouse suggests that the large Dystrophin isoforms may also influence neurotransmitter release at other types of synapses (Green et al., 2004). Whether the CNS-specific Dp186 isoform plays roles at Drosophila interneuronal synapses, similar to those played by DLP2 at the NMJ, remains to be evaluated. Disrupted homeostasis at Dystrophindeficient interneuronal brain synapses might contribute to the poorly understood mental impairments associated with DMD in humans.

\section{References}

Aberle H, Haghighi AP, Fetter RD, McCabe BD, Magalhaes TR, Goodman CS (2002) Wishful thinking encodes a BMP type II receptor that regulates synaptic growth in Drosophila. Neuron 33:545-558.

Atwood HL, Govind CK, Wu CF (1993) Differential ultrastructure of synaptic terminals on ventral longitudinal abdominal muscles in Drosophila larvae. J Neurobiol 24:1008-1024.

Baines RA (2004) Synaptic strengthening mediated by bone morphogenetic protein-dependent retrograde signaling in the Drosophila CNS. J Neurosci 24:6904-6911.

Blake DJ, Weir A, Newey SE, Davies KE (2002) Function and genetics of dystrophin and dystrophin-related proteins in muscle. Physiol Rev 82:291-329.

Boyd IA, Martin AR (1956) The end-plate potential in mammalian muscle. J Physiol (Lond) 132:74-91.

Brand AH, Perrimon N (1993) Targeted gene expression as a means of altering cell fates and generating dominant phenotypes. Development 118:401-415.

Carlson CG (1998) The dystrophinopathies: an alternative to the structural hypothesis. Neurobiol Dis 5:3-15.

Cull-Candy SG, Miledi R, Trautmann A, Uchitel OD (1980) On the release of transmitter at normal, myasthenia gravis and myasthenic syndrome affected human end-plates. J Physiol (Lond) 299:621-638.

Davis GW, Bezprozvanny I (2001) Maintaining the stability of neural function: a homeostatic hypothesis. Annu Rev Physiol 63:847-869.

Davis GW, DiAntonio A, Petersen SA, Goodman CS (1998) Postsynaptic PKA controls quantal size and reveals a retrograde signal that regulates presynaptic transmitter release in Drosophila. Neuron 20:305-315.

Deconinck AE, Rafael JA, Skinner JA, Brown SC, Potter AC, Metzinger L, Watt DJ, Dickson JG, Tinsley JM, Davies KE (1997a) Utrophindystrophin-deficient mice as a model for Duchenne muscular dystrophy. Cell 90:717-727.

Deconinck AE, Potter AC, Tinsley JM, Wood SJ, Vater R, Young C, Metzinger L, Vincent A, Slater CR, Davies KE (1997b) Postsynaptic abnormalities at the neuromuscular junctions of utrophin-deficient mice. J Cell Biol 136:883-894.

Dekkers LC, van der Plas MC, van Loenen PB, den Dunnen JT, van Ommen GJ, Fradkin LG, Noordermeer JN (2004) Embryonic expression patterns of the Drosophila dystrophin-associated glycoprotein complex orthologs. Gene Expr Patterns 4:153-159.

DiAntonio A, Petersen SA, Heckmann M, Goodman CS (1999) Glutamate receptor expression regulates quantal size and quantal content at the Drosophila neuromuscular junction. J Neurosci 19:3023-3032.

Ervasti JM, Campbell KP (1991) Membrane organization of the dystrophinglycoprotein complex. Cell 66:1121-1131.

Gailly P (2002) New aspects of calcium signaling in skeletal muscle cells: implications in Duchenne muscular dystrophy. Biochim Biophys Acta 1600:38-44.

Grady RM, Teng H, Nichol MC, Cunningham JC, Wilkinson RS, Sanes JR (1997) Skeletal and cardiac myopathies in mice lacking utrophin and dystrophin: a model for Duchenne muscular dystrophy. Cell 90:729-738.

Green DG, Guo H, Pillers DA (2004) Normal photoresponses and altered b-wave responses to APB in the $\mathrm{mdx}(\mathrm{Cv} 3)$ mouse isolated retina ERG supports role for dystrophin in synaptic transmission. Vis Neurosci 21:739-747.

Greener MJ, Roberts RG (2000) Conservation of components of the dystrophin complex in Drosophila. FEBS Lett 482:13-18.

Griffith LC, Verselis LM, Aitken KM, Kyriacou CP, Danho W, Greenspan RJ (1993) Inhibition of calcium/calmodulin-dependent protein kinase in Drosophila disrupts behavioral plasticity. Neuron 10:501-509.

Grinnell AD, Chen BM, Kashani A, Lin J, Suzuki K, Kidokoro Y (2003) The role of integrins in the modulation of neurotransmitter release from motor nerve terminals by stretch and hypertonicity. J Neurocytol 32:489-503.

Haghighi AP, McCabe BD, Fetter RD, Palmer JE, Hom S, Goodman CS (2003) Retrograde control of synaptic transmission by postsynaptic CaMKII at the Drosophila neuromuscular junction. Neuron 39:255-267.

Hoffman EP, Brown Jr RH, Kunkel LM (1987) Dystrophin: the protein product of the Duchenne muscular dystrophy locus. Cell 51:919-928.

Jan LY, Jan YN (1976) L-Glutamate as an excitatory transmitter at the Drosophila larval neuromuscular junction. J Physiol (Lond) 262:215-236.

Jan LY, Jan YN (1982) Antibodies to horseradish peroxidase as specific neuronal markers in Drosophila and in grasshopper embryos. Proc Natl Acad Sci USA 79:2700-2704.

Jia XX, Gorczyca M, Budnik V (1993) Ultrastructure of neuromuscular junctions in Drosophila: comparison of wild type and mutants with increased excitability. J Neurobiol 24:1025-1044.

Jin P, Griffith LC, Murphey RK (1998) Presynaptic calcium/calmodulindependent protein kinase II regulates habituation of a simple reflex in adult Drosophila. J Neurosci 18:8955-8964.

Johnson BD, Scheuer T, Catterall WA (2005) Convergent regulation of skeletal muscle $\mathrm{Ca}^{2+}$ channels by dystrophin, the actin cytoskeleton, and cAMP-dependent protein kinase. Proc Natl Acad Sci USA 102:4191-4196.

Keshishian H, Kim YS (2004) Orchestrating development and function: retrograde BMP signaling in the Drosophila nervous system. Trends Neurosci 27:143-147.

Kidokoro Y, Kuromi H, Delgado R, Maureira C, Oliva C, Labarca P (2004) Synaptic vesicle pools and plasticity of synaptic transmission at the Drosophila synapse. Brain Res Brain Res Rev 47:18-32.

Kim H, Rogers MJ, Richmond JE, McIntire SL (2004) SNF-6 is an acetylcholine transporter interacting with the dystrophin complex in Caenorhabditis elegans. Nature 430:891-896.

Lin DM, Goodman CS (1994) Ectopic and increased expression of Fasciclin II alters motoneuron growth cone guidance. Neuron 13:507-523.

Luo L, Liao YJ, Jan LY, Jan YN (1994) Distinct morphogenetic functions of similar small GTPases: Drosophila Drac1 is involved in axonal outgrowth and myoblast fusion. Genes Dev 8:1787-1802.

Lyons PR, Slater CR (1991) Structure and function of the neuromuscular junction in young adult mdx mice. J Neurocytol 20:969-981.

Madhavan R, Massom LR, Jarrett HW (1992) Calmodulin specifically binds three proteins of the dystrophin-glycoprotein complex. Biochem Biophys Res Commun 185:753-759.

Marques G, Bao H, Haerry TE, Shimell MJ, Duchek P, Zhang B, O'Connor MB (2002) The Drosophila BMP type II receptor Wishful Thinking reg- 
ulates neuromuscular synapse morphology and function. Neuron 33:529-543.

Marrus SB, Portman SL, Allen MJ, Moffat KG, DiAntonio A (2004) Differential localization of glutamate receptor subunits at the Drosophila neuromuscular junction. J Neurosci 24:1406-1415.

Martin AR (1955) A further study of the statistical composition on the endplate potential. J Physiol (Lond) 130:114-122.

Martin AR (1966) Quantal nature of synaptic transmission. Physiol Rev 46:51-66.

McCabe BD, Marques G, Haghighi AP, Fetter RD, Crotty ML, Haerry TE, Goodman CS, O'Connor MB (2003) The BMP homolog Gbb provides a retrograde signal that regulates synaptic growth at the Drosophila neuromuscular junction. Neuron 39:241-254.

Megeney LA, Kablar B, Garrett K, Anderson JE, Rudnicki MA (1996) MyoD is required for myogenic stem cell function in adult skeletal muscle. Genes Dev 10:1173-1183.

Neuman S, Kaban A, Volk T, Yaffe D, Nudel U (2001) The dystrophin/ utrophin homologues in Drosophila and in sea urchin. Gene 263:17-29.

Paradis S, Sweeney ST, Davis GW (2001) Homeostatic control of presynaptic release is triggered by postsynaptic membrane depolarization. Neuron 30:737-749.

Parnas D, Haghighi AP, Fetter RD, Kim SW, Goodman CS (2001) Regulation of postsynaptic structure and protein localization by the Rho-type guanine nucleotide exchange factor dPix. Neuron 32:415-424.

Petersen SA, Fetter RD, Noordermeer JN, Goodman CS, DiAntonio A (1997) Genetic analysis of glutamate receptors in Drosophila reveals a retrograde signal regulating presynaptic transmitter release. Neuron 19:1237-1248.

Plomp JJ, van Kempen GT, Molenaar PC (1992) Adaptation of quantal content to decreased postsynaptic sensitivity at single endplates in alphabungarotoxin-treated rats. J Physiol (Lond) 458:487-499.

Plomp JJ, Van Kempen GT, De Baets MB, Graus YM, Kuks JB, Molenaar PC (1995) Acetylcholine release in myasthenia gravis: regulation at single end-plate level. Ann Neurol 37:627-636.

Rando TA (2001) The dystrophin-glycoprotein complex, cellular signaling, and the regulation of cell survival in the muscular dystrophies. Muscle Nerve 24:1575-1594.

Razzaq A, Robinson IM, McMahon HT, Skepper JN, Su Y, Zelhof AC, Jackson AP, Gay NJ, O'Kane CJ (2001) Amphiphysin is necessary for organization of the excitation-contraction coupling machinery of muscles, but not for synaptic vesicle endocytosis in Drosophila. Genes Dev 15:2967-2979.

Reichhart JM, Ligoxygakis P, Naitza S, Woerfel G, Imler JL, Gubb D (2002) Splice-activated UAS hairpin vector gives complete RNAi knockout of single or double target transcripts in Drosophila melanogaster. Genesis 34:160-164.
Rohrbough J, Pinto S, Mihalek RM, Tully T, Broadie K (1999) latheo, a Drosophila gene involved in learning, regulates functional synaptic plasticity. Neuron 23:55-70.

Rorth P (1996) A modular misexpression screen in Drosophila detecting tissue-specific phenotypes. Proc Natl Acad Sci USA 93:12418-12422.

Sandrock Jr AW, Dryer SE, Rosen KM, Gozani SN, Kramer R, Theill LE, Fischbach GD (1997) Maintenance of acetylcholine receptor number by neuregulins at the neuromuscular junction in vivo. Science 276:599-603.

Sandstrom DJ (2004) Isoflurane depresses glutamate release by reducing neuronal excitability at the Drosophila neuromuscular junction. J Physiol (Lond) 558:489-502.

Sanyal S, Kim SM, Ramaswami M (2004) Retrograde regulation in the CNS; neuron-specific interpretations of TGF-beta signaling. Neuron 41:845-848.

Schulz PE, Cook EP, Johnston D (1994) Changes in paired-pulse facilitation suggest presynaptic involvement in long-term potentiation. J Neurosci 14:5325-5337.

Schuster CM, Ultsch A, Schloss P, Cox JA, Schmitt B, Betz H (1991) Molecular cloning of an invertebrate glutamate receptor subunit expressed in Drosophila muscle. Science 254:112-114.

Segalat L (2002) Dystrophin and functionally related proteins in the nematode Caenorhabditis elegans. Neuromuscul Disord 12 [Suppl 1]:S105-S109.

Stevens CF, Tsujimoto T (1995) Estimates for the pool size of releasable quanta at a single central synapse and for the time required to refill the pool. Proc Natl Acad Sci USA 92:846-849.

Suzuki K, Okamoto T, Kidokoro Y (2002) Biphasic modulation of synaptic transmission by hypertonicity at the embryonic Drosophila neuromuscular junction. J Physiol (Lond) 545:119-131.

Tanimoto H, Itoh S, ten Dijke P, Tabata T (2000) Hedgehog creates a gradient of DPP activity in Drosophila wing imaginal discs. Mol Cell 5:59-71.

Toba G, Ohsako T, Miyata N, Ohtsuka T, Seong KH, Aigaki T (1999) The gene search system. A method for efficient detection and rapid molecular identification of genes in Drosophila melanogaster. Genetics 151:725-737.

Tower J, Karpen GH, Craig N, Spradling AC (1993) Preferential transposition of Drosophila P elements to nearby chromosomal sites. Genetics 133:347-359.

Turrigiano GG (1999) Homeostatic plasticity in neuronal networks: the more things change, the more they stay the same. Trends Neurosci 22:221-227.

Watkins SC, Hoffman EP, Slayter HS, Kunkel LM (1988) Immunoelectron microscopic localization of dystrophin in myofibres. Nature 333:863-866.

Zucker RS, Regehr WG (2002) Short-term synaptic plasticity. Annu Rev Physiol 64:355-405. 